\author{
N. Katakami $\cdot$ H. Kaneto $\cdot$ M. Matsuhisa $\cdot$ \\ T. Miyatsuka - K. Sakamoto - D. Kawamori • \\ K. Yoshiuchi $\cdot$ Y. Nakamura $\cdot$ K. Kato $\cdot$ K. Yamamoto $\cdot$ \\ K. Kosugi · M. Hori · Y. Yamasaki
}

\title{
Association of soluble CD40 ligand with carotid atherosclerosis in Japanese type 1 diabetic patients
}

Received: 24 November 2005 / Accepted: 16 March 2006 / Published online: 12 May 2006

(C) Springer-Verlag 2006

\begin{abstract}
Aims/hypothesis: It has recently been shown that the soluble form of CD40 ligand (sCD40L) interacts with CD40 on vascular cells, leading to a variety of proinflammatory responses, and that serum sCD40L levels can be a predictive marker of cardiovascular events. The aim of this study was to estimate SCD40L levels in type 1 diabetic patients to examine a possible association with carotid atherosclerosis. Subjects and methods: Human sCD40L levels in serum and intima-media thickness (IMT) of carotid artery were examined in 80 Japanese type 1 diabetic patients $(27$ men and 53 women, age $22.8 \pm$ 3.4 years (mean $\pm \mathrm{SD}$ ), duration of diabetes $13.2 \pm 6.1$ years) and 20 healthy age-matched non-diabetic individuals. Results: Serum sCD40L levels were significantly $(p=0.0185)$ higher in subjects with type 1 diabetes $(2.10 \pm$ $1.33 \mathrm{ng} / \mathrm{ml})$ compared with non-diabetic subjects $(1.35 \pm$ $0.88 \mathrm{ng} / \mathrm{ml}$ ). The greatest IMT (Max-IMT) and averaged IMT (Mean-IMT) were also significantly greater in patients with type 1 diabetes than in control subjects $(0.73 \pm 0.14$ vs $0.64 \pm 0.07 \mathrm{~mm}, p=0.0041,0.63 \pm 0.09$ vs $0.57 \pm 0.06 \mathrm{~mm}$, $p=0.0066$, respectively). Levels of sCD40L were statistically significantly associated with Max-IMT $(r=0.383$, $p<0.001)$ and Mean-IMT $(r=0.275, p=0.0058)$. Furthermore, stepwise multivariate regression analyses demonstrated that SCD40L is a determinant of both Max- and Mean-IMT, independently of conventional risk
\end{abstract}

N. Katakami $(\bowtie) \cdot$ H. Kaneto $\cdot$ M. Matsuhisa $\cdot$ T. Miyatsuka $\cdot$ K. Sakamoto - D. Kawamori · K. Yoshiuchi · Y. Nakamura · K. Kato $\cdot$ K. Yamamoto $\cdot$ M. Hori · Y. Yamasaki Department of Internal Medicine and Therapeutics (A8) Osaka University Graduate School of Medicine, 2-2, Yamadaoka,

Suita, Osaka 565-0871, Japan

e-mail: katakami@medone.med.osaka-u.ac.jp

Tel.: +81-6-68793633

Fax: +81-6-68793639

K. Kosugi

Department of Internal Medicine

Osaka Police Hospital,

Osaka, Japan factors. Conclusions/interpretation: It is suggested that increased levels of serum sCD40L are associated with accelerated atherosclerotic change observed in young patients with type 1 diabetes.

Keywords Atherosclerosis - Carotid artery - CD40 ligand - IMT · Intima-media thickness - Type 1 diabetes

Abbreviations hs-CRP: high-sensitivity C-reactive protein - IMT: intima-media thickness - $\mathrm{sCD} 40 \mathrm{~L}$ : soluble CD40 ligand

\section{Introduction}

Platelet hyperactivity plays an important role in the pathogenesis of atherosclerosis. Although CD40 ligand (CD40L) was originally identified on $\mathrm{CD}^{+} \mathrm{T}$ cells, it has recently been found on activated platelets. Membranebound CD40L interacts with CD40 on vascular cells, which leads to a variety of proinflammatory and prothrombotic responses, including the release of cytokines and induction of adhesion molecule expression [1]. Adhesion molecules mediate leucocyte adhesion to the endothelium and transendothelial migration, which is an important early step in the atherosclerotic process $[2,3]$. Furthermore, disruption of CD40-CD40L interactions reduces atherosclerotic lesions in hypercholesterolaemic mice $[4,5]$. The soluble form of CD40L (sCD40L) is similar to membrane-bound CD40L in terms of its actions on monocytes and endothelial cells, and is involved in inflammation and thrombosis [6, 7]. It has been suggested that increased levels of $\mathrm{sCD} 40 \mathrm{~L}$ are associated with atherosclerotic diseases [8-10] and can be a predictive marker of cardiovascular events [11, 12].

In type 1 diabetic patients, atherosclerosis is often observed clinically and platelet activity is thought to be increased [13-15]. It has not yet been fully evaluated, however, whether the platelet hyperactivity is actually associated with the progression of atherosclerosis in this group of patients. Thus, the aim of this study, was to 
examine whether platelet hyperactivity indicated by serum sCD40L levels is associated with carotid atherosclerosis in type 1 diabetic patients.

\section{Subjects and methods}

Study population A total of 80 Japanese type 1 diabetic patients ( 27 men and 53 women, age $22.8 \pm 3.4$ years (mean \pm $\mathrm{SD}$ ), duration of diabetes $13.2 \pm 6.1$ years, daily net urinary C-peptide $1.8 \pm 2.9 \mathrm{nmol} /$ day [5.3 $\pm 8.7 \mu \mathrm{g} / \mathrm{day}]$ ) undergoing periodic follow-up examinations at the Diabetes Clinic of Osaka University Hospital and the Osaka Police Hospital were enrolled in this study. All diabetic patients were treated with at least three or four daily insulin injections or a continuous subcutaneous insulin infusion. The daily insulin dose was $0.88 \pm 0.26 \mathrm{U} / \mathrm{kg}$. We enrolled 20 age-matched healthy non-diabetic individuals ( 9 men and 11 women, age $24.2 \pm 1.5$ years) as control subjects. The subjects did not have any clinical evidence of infection, connective tissue disease, liver dysfunction or angiopathy. None of the subjects received antihypertensive, antiplatelet or lipidlowering medication during this study. After a full explanation of the study, which was approved by the Ethical Committee for Human Studies at Osaka University Graduate School of Medicine, written informed consent was obtained from each subject.

Laboratory procedures Fasting blood samples were collected and the following laboratory analyses were performed by SRL (Tokyo, Japan). Serum total cholesterol and HDL cholesterol, serum triglyceride and $\mathrm{HbA}_{1 \mathrm{c}}$ levels were measured using standard laboratory protocols. LDL cholesterol was calculated using the Friedewald formula [16]. Serum high-sensitivity C-reactive protein (hs-CRP) was measured by ELISA. BMI, systolic BP and diastolic $\mathrm{BP}$ were also measured.

The subjects submitted urine samples that had been collected at home over the previous $24 \mathrm{~h}$. Written instructions and careful explanation regarding the procedure for urine collection were given to each subject. Most of the patients with diabetes were familiar with the method for collecting urine at home. Nevertheless, a urine sample was discarded if there was any doubt with regard to its collection. Each participant's daily urinary AER was calculated by multiplying their 24-h urine volume by their urinary albumin concentration measured using standard laboratory protocols. Patients were classified according to urinary AER into the normoalbuminuria group (urinary $\mathrm{AER}<30 \mathrm{mg} / \mathrm{day}, n=68$ ), microalbuminuria group (urinary AER 30-300 mg/day, $n=7$ ) or the macroalbuminuria group (urinary AER $>300 \mathrm{mg} /$ day, $n=1$ ). The mean urinary AER was $18.3 \pm 43.1 \mathrm{mg} /$ day. Presence of retinopathy was diagnosed by ophthalmologists based on the findings of funduscopy; there were 53 patients without retinopathy, 21 with simple retinopathy and four with proliferative retinopathy.

Measurement of serum levels of $s C D 40 L$ The BioSource Human sCD40L Immunoassay Kit (Camarillo, CA, USA) was used to assess serum sCD40L levels. Measurements were performed according to the manufacturer's instruc-

Table 1 Baseline characteristics

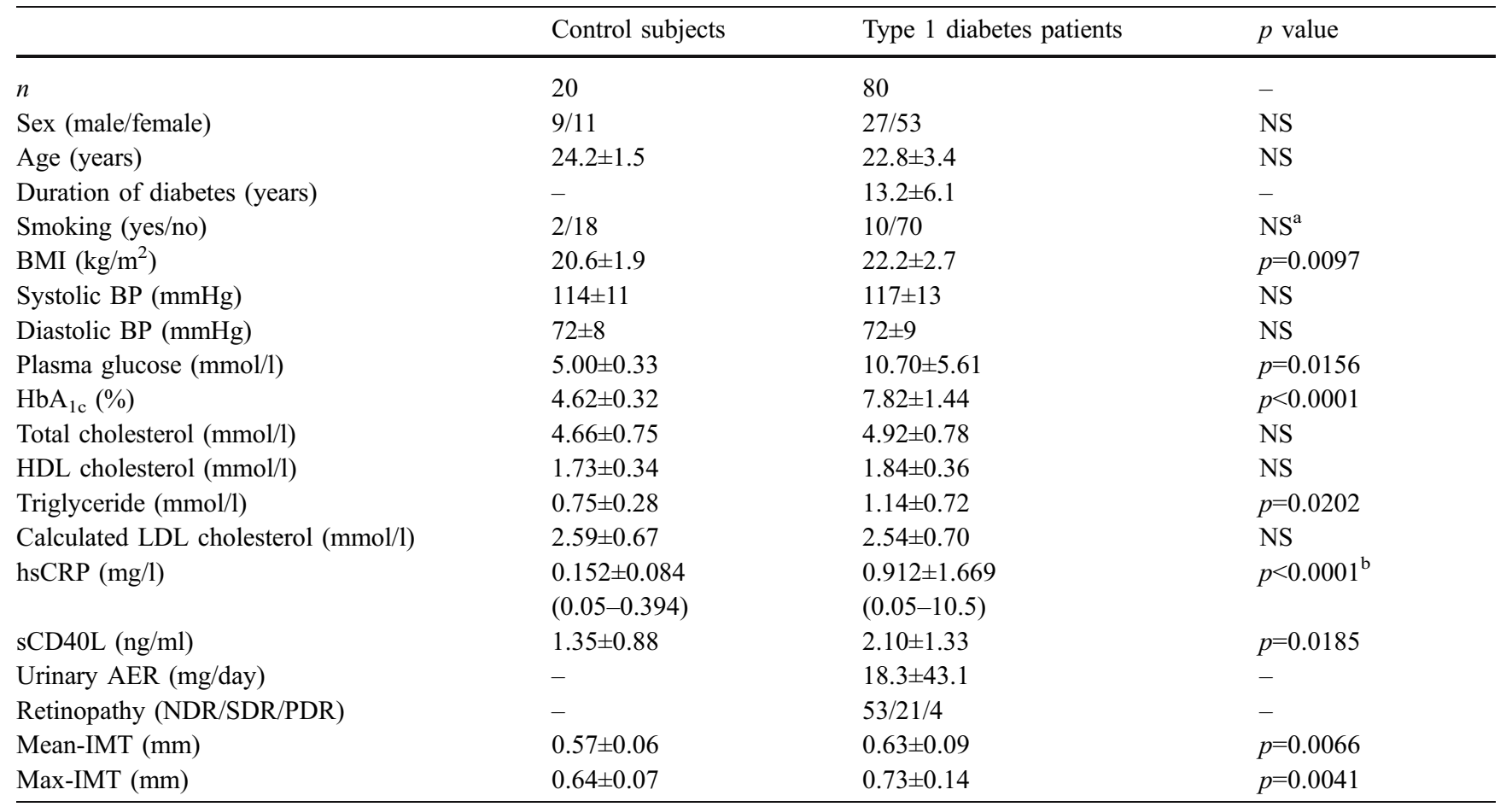

Data are means \pm SD. The Student's $t$-test was performed. ${ }^{\mathrm{a}} \chi^{2}$ test; ${ }^{\mathrm{b}}$ Mann-Whitney $U$-test

$N D R$ No diabetic retinopathy, $S D R$ simple diabetic retinopathy, $P D R$ proliferative diabetic retinopathy 


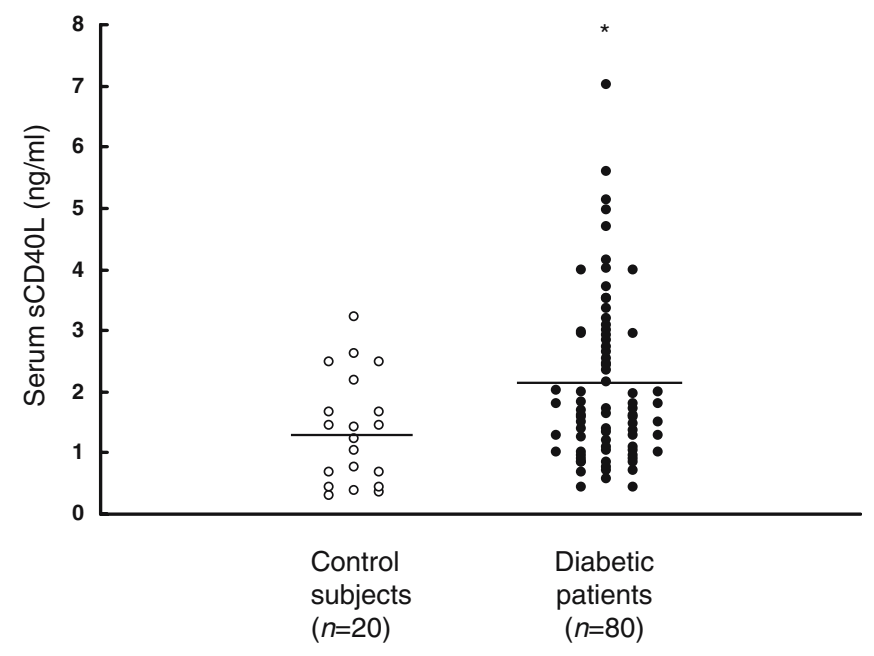

Fig. 1 Serum levels of sCD40L were significantly $(p=0.0185)$ higher in subjects with type 1 diabetes $(2.10 \pm 1.33 \mathrm{ng} / \mathrm{ml})$ than in age-matched healthy control subjects $(1.35 \pm 0.88 \mathrm{ng} / \mathrm{ml}) .{ }^{*} p=0.0185$ vs control subjects

tions. The intra-assay CV was $4.0 \%$.

Measurement of IMT To identify early stages of atherosclerosis, ultrasonographic scanning of the carotid artery was performed using an echotomographic system (SSA340A, Toshiba, Tokyo, Japan) with an electrical linear transducer (midfrequency $7.5 \mathrm{MHz}$ ). Scanning of the extracranial common carotid artery, the carotid bulb and the internal carotid artery in the neck was performed bilaterally from three different longitudinal projections (i.e. anterior oblique, lateral and posterior oblique), as well as the transverse projections, as reported in our previous studies [15-18]. All of the images were photocopied. The detection limit of this echo system, using 7.5 MHz, was
$0.1 \mathrm{~mm}$. The IMT was measured as the distance from the leading edge of the first echogenic line to the leading edge of the second echogenic line [19]. The first line represents the lumen-intima interface, and the second line is produced by the collagen-containing upper layer of the tunica adventitia. At each longitudinal projection, the site of the greatest thickness including a plaque lesion was sought along the arterial walls. Three determinations of IMT were conducted at the site of the thickest pointmaximum IMT (Max-IMT) - and two adjacent points (located $1 \mathrm{~cm}$ upstream and $1 \mathrm{~cm}$ downstream from this site). These three determinations were averaged (MeanIMT). The greatest value among the six Max- or MeanIMTs (three from the left and three from right) was used as the representative value for each individual. All ultrasound scans were performed by an experienced sonographer $(\mathrm{K}$. Sakamoto), and an experienced physician (N. Katakami) determined IMT from the photograph; both were unaware of the subject's study group and clinical characteristics. Reproducibility of the Mean-IMT measurement was examined 1 week later in 30 participants with type 1 diabetes by the same sonographer and the same physician. The mean difference in mean-IMT between these two determinations was $0.04 \mathrm{~mm}$ and the standard deviation was $0.07 \mathrm{~mm}$, demonstrating good reproducibility for repeated measurements.

Statistical analysis Data are given as means \pm SD. Means or proportions for clinical characteristics were computed for the case and control subjects, and the laboratory data were compared using unpaired $t$-tests. Differences in proportions were tested using the $\chi^{2}$ test. Because the CRP distributions were skewed to the left, the significance of any differences between the patients and control subjects was determined using the Mann-Whitney $U$-test. For the

Table 2 Correlation between sCD40L and variables in the subjects

\begin{tabular}{|c|c|c|c|c|c|}
\hline & \multicolumn{2}{|c|}{ Univariate analysis $^{\mathrm{a}}$} & \multicolumn{3}{|c|}{ Multivariate analysis } \\
\hline & $r$ value & $p$ value & $\beta$ coefficient & $F$ value & $p$ value \\
\hline Age (years) & -0.070 & NS & NI & 0.1 & - \\
\hline Sex (male/female) & - & NS & NI & 1.1 & - \\
\hline Duration of diabetes (years) & -0.023 & NS & $\mathrm{NI}$ & 0.1 & - \\
\hline Smoking (yes/no) & -0.043 & NS & NI & 0.1 & - \\
\hline BMI $\left(\mathrm{kg} / \mathrm{m}^{2}\right)$ & 0.190 & NS & NI & 0.6 & - \\
\hline Systolic BP (mmHg) & 0.140 & NS & NI & 0.6 & - \\
\hline Diastolic BP (mmHg) & 0.223 & 0.0269 & NI & 2.0 & - \\
\hline Plasma glucose $(\mathrm{mmol} / \mathrm{l})$ & 0.058 & NS & $\mathrm{NI}$ & 0.1 & - \\
\hline $\mathrm{HbA}_{1 \mathrm{c}}(\%)$ & 0.117 & NS & $\mathrm{NI}$ & 0.1 & - \\
\hline Total cholesterol (mmol/l) & 0.008 & NS & NI & 0.1 & - \\
\hline HDL cholesterol (mmol/l) & 0.018 & NS & NI & 0.0 & - \\
\hline Triglyceride (mmol/l) & 0.072 & NS & $\mathrm{NI}$ & 0.4 & - \\
\hline LDL cholesterol (mmol/l) & -0.051 & NS & $\mathrm{NI}$ & 1.0 & - \\
\hline $\log _{10} \mathrm{hs}-\mathrm{CRP}(\mathrm{mg} / \mathrm{l})$ & 0.285 & 0.0043 & 0.684 & 8.1 & 0.006 \\
\hline$R^{2}$ & - & - & 0.078 & - & - \\
\hline
\end{tabular}

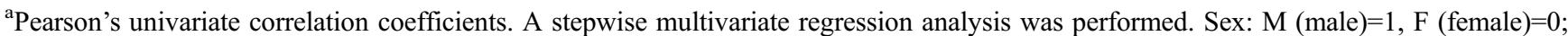
Smoking: yes $=1$, no $=0$

$\beta$ Partial regression coefficient, $N I$ not included in the model because variable was not significant 
forward and backward stepwise multivariate regression analyses, the $F$ value for the inclusion and exclusion of variables was set at 4.0. These statistical analyses were performed using Stat-View statistical software (Version 5.0 for Windows; Abacus Concepts, Berkeley, CA, USA) and HALBOU statistical software (Gendai Sugaku-sha, Kyoto, Japan) on a personal computer. The threshold of statistical significance was defined as $p<0.05$.

\section{Results}

Serum levels of $s C D 40 L$ are higher in type 1 diabetic patients than in non-diabetic subjects The clinical and biochemical characteristics of the study subjects are presented in Table 1. BMI, plasma glucose, $\mathrm{HbA}_{1 \mathrm{c}}$, serum triglyceride, and hs-CRP levels were significantly higher in subjects with type 1 diabetes than in non-diabetic subjects $(p<0.05)$. There was no significant difference between the two groups regarding the other clinical parameters, such as age, sex, current smoking habit, sBP, dBP, total cholesterol, LDL cholesterol and HDL cholesterol.

Levels of sCD40L were significantly $(p=0.0185)$ higher in subjects with type 1 diabetes compared with nondiabetic subjects $(2.10 \pm 1.33$ vs $1.35 \pm 0.88 \mathrm{ng} / \mathrm{ml}$, respectively) (Fig. 1). Although mean overall sCD40L levels (20 control subjects, 80 diabetic patients) were correlated with dBP $(r=0.223, p=0.0269)$ and common logarithm of hsCRP $(r=0.285, p=0.0043)$, sCD40L levels were not significantly correlated with plasma glucose, $\mathrm{HbA}_{1 \mathrm{c}}$, duration of diabetes or LDL cholesterol levels (Table 2). When only the diabetic subjects were analysed, there were still statistically significant correlations between sCD40L and diastolic BP $(r=0.276, p=0.0141)$ or $\log _{10}$ hs-CRP $(r=0.249, p=0.0275)$. Furthermore, a stepwise multivariate regression analysis including age, duration of diabetes, sex, current smoking habit, BMI, systolic BP, diastolic BP, plasma glucose, $\mathrm{HbA}_{1 \mathrm{c}}$, total cholesterol, HDL cholesterol, LDL cholesterol, triglyceride and $\log _{10}$ hs-CRP revealed that $\log _{10}$ hs-CRP $(F=8.1, p=0.006)$ was an independent determinant of a high $\mathrm{SCD} 40 \mathrm{~L}$ value (Table 2). Additionally, we performed a stepwise multivariate regression analysis including the existence of type 1 diabetes, sex, BMI, plasma glucose, $\mathrm{HbA}_{1 \mathrm{c}}$ and triglyceride as independent variables, and sCD40L levels as an induced variable. This analysis demonstrated that type 1 diabetes is the independent determinant $(F=5.4$, $p=0.0228$ ) for high sCD40L levels, suggesting that the presence of type 1 diabetes affects SCD40L levels independently of sex and BMI.

Univariate regression analysis also revealed that $\log _{10}$ hs-CRP was significantly correlated with BMI $(r=0.478$, $p<0.0001), \quad \operatorname{sBP}(r=0.375, p=0.0004), \quad \mathrm{dBP} \quad(r=0.480$, $p<0.0001), \mathrm{HbA}_{1 \mathrm{c}}(r=0.268, p=0.0140)$ and total cholesterol $(r=0.246, \quad p=0.0245)$ but not with the other parameters, including duration of diabetes. A stepwise multivariate regression analysis demonstrated that BMI $(F=6.6)$ and dBP $(F=7.2)$ were the independent determi- nants for a high $\log _{10}$ hs-CRP.

Association between SCD40L and carotid IMT Mean-IMT and Max-IMT were significantly greater in patients with type 1 diabetes than in control subjects $(0.63 \pm 0.09$ vs $0.57 \pm 0.06 \mathrm{~mm}, p=0.0066 ; 0.73 \pm 0.14$ vs $0.64 \pm 0.07 \mathrm{~mm}$, $p=0.0041$, respectively) (Table 1 ). Serum sCD40L was statistically significantly associated with Mean-IMT $(r=0.275, p=0.0058)$ and Max-IMT $(r=0.383, p<0.001)$ (Fig. 2). Mean-IMT was also correlated with BMI $(r=0.279, p=0.0047), \quad \mathrm{sBP} \quad(r=0.235, p=0.0176) \quad$ and $\log _{10}$ hs-CRP $(r=0.334, p=0.0006)$. Max-IMT was also correlated with BMI $(r=0.25, p=0.0119)$ and $\mathrm{sBP}$ $(r=0.287, p=0.0035)$ (Table 3$)$. However, plasma glucose levels and calculated LDL cholesterol levels were not correlated with Mean-IMT or Max-IMT. Analysis of the diabetic subjects alone showed statistically significant correlations between sCD40L and Mean-IMT $(r=0.277$, $p=0.0139)$ and $\operatorname{Max}-\operatorname{IMT}(r=0.376, p=0.006)$. To demonstrate that $\mathrm{sCD} 40 \mathrm{~L}$ is an independent determinant of Mean-IMT, we performed a stepwise multivariate regres-
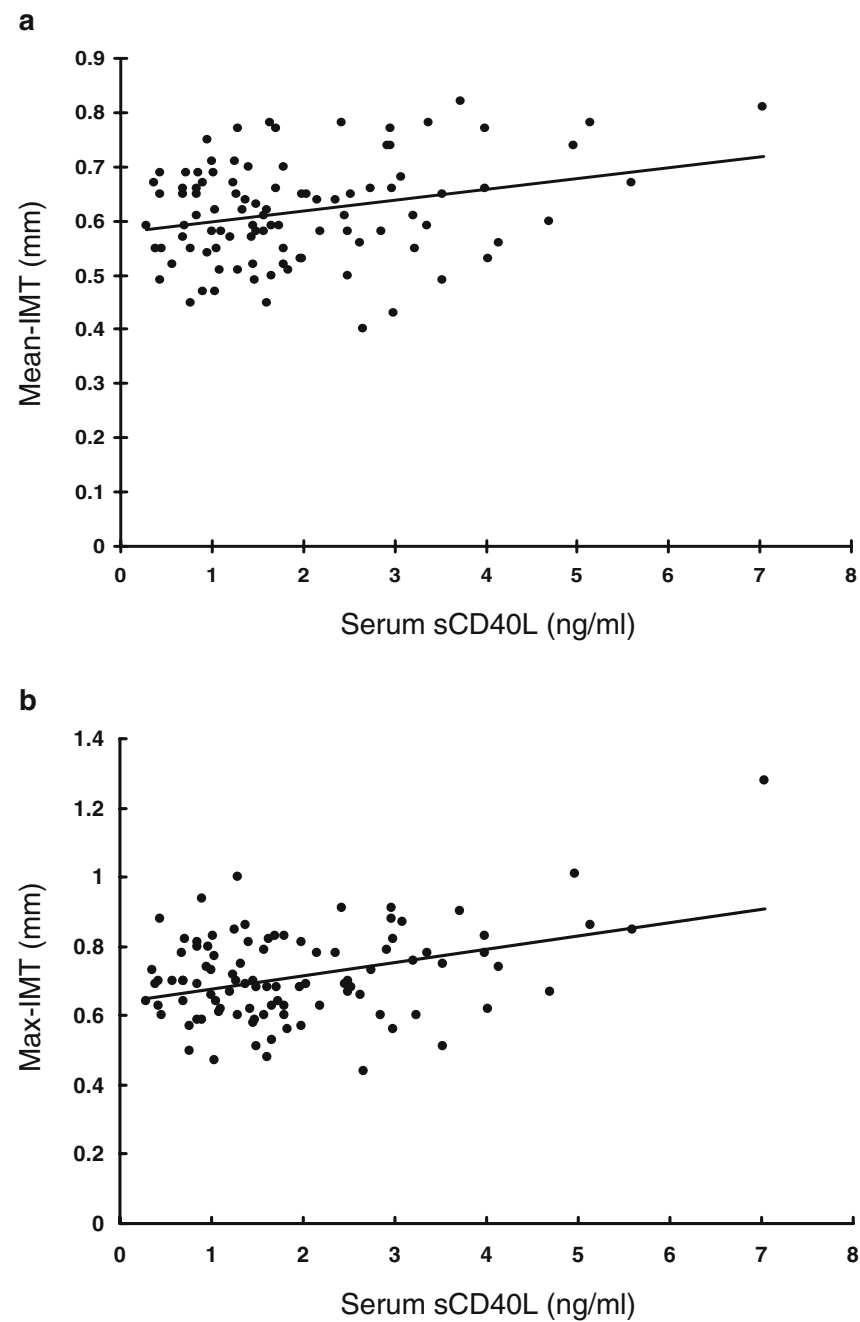

Fig. 2 Levels of sSC40L showed statistically significant associations with (a) Mean-IMT $(r=0.275, p=0.0058)$ and (b) Max-IMT $(r=0.383, p<0.001)$ 
Table 3 Correlation between IMT and variables in the subjects

\begin{tabular}{|c|c|c|c|c|c|c|c|c|c|c|}
\hline & \multicolumn{5}{|c|}{ Mean-IMT } & \multicolumn{5}{|c|}{ Max-IMT } \\
\hline & \multicolumn{2}{|c|}{ Univariate analysis $^{\mathrm{a}}$} & \multicolumn{3}{|c|}{ Multivariate analysis } & \multicolumn{2}{|c|}{ Univariate analysis $^{\mathrm{a}}$} & \multicolumn{3}{|c|}{ Multivariate analysis } \\
\hline & $r$ value & $p$ value & $\beta$ coefficient & $F$ value & $p$ value & $r$ value & $p$ value & $\begin{array}{l}\beta \\
\text { coefficient }\end{array}$ & $F$ value & $p$ value \\
\hline Age (years) & 0.051 & NS & NI & 1.5 & - & 0.134 & NS & NI & 2.3 & - \\
\hline Sex (male/female) & - & NS & NI & 3.1 & - & - & 0.0137 & NI & 1.9 & - \\
\hline $\operatorname{BMI}\left(\mathrm{kg} / \mathrm{m}^{2}\right)$ & 0.279 & 0.0047 & NI & 1.6 & - & 0.250 & 0.0119 & NI & 0.8 & - \\
\hline Systolic BP (mmHg) & 0.235 & 0.0176 & NI & 3.1 & - & 0.287 & 0.0035 & 0.002 & 6.8 & 0.011 \\
\hline Diastolic BP (mmHg) & 0.189 & NS & NI & 0.7 & - & 0.176 & NS & $\mathrm{NI}$ & 0.5 & - \\
\hline Plasma glucose $(\mathrm{mmol} / \mathrm{l})$ & 0.135 & NS & NI & 0.6 & & 0.157 & NS & $\mathrm{NI}$ & 0.4 & - \\
\hline $\mathrm{HbA}_{1 \mathrm{c}}(\%)$ & 0.042 & NS & NI & 1.2 & - & 0.041 & NS & NI & 0.0 & - \\
\hline LDL cholesterol (mmol/l) & 0.171 & NS & NI & 0.0 & - & 0.149 & NS & $\mathrm{NI}$ & 0.7 & - \\
\hline $\log _{10}$ CRP $(\mathrm{mg} / \mathrm{l})$ & 0.334 & 0.0006 & 0.046 & 6.8 & 0.011 & 0.159 & NS & NI & 0.1 & - \\
\hline sCD40L (ng/ml) & 0.275 & 0.0058 & 0.014 & 4.3 & 0.041 & 0.383 & $<0.001$ & 0.035 & 13.6 & $<0.001$ \\
\hline$R^{2}$ & - & - & 0.141 & - & - & - & - & 0.203 & - & - \\
\hline
\end{tabular}

aPearson's univariate correlation coefficients. A stepwise multivariate regression analysis was performed. Sex: M (male)=1, F (female) $=0$; Smoking: yes $=1$, no $=0$

$\beta$ Partial regression coefficient, $N I$ not included in the model because variable was not significant

sion analysis including age, duration of diabetes, sex, current smoking habit, BMI, systolic BP, diastolic BP, plasma glucose, $\mathrm{HbA}_{1 \mathrm{c}}$, total cholesterol, HDL cholesterol, LDL cholesterol, triglyceride and $\log _{10}$ hs-CRP as independent variables and found that $\mathrm{sCD} 40 \mathrm{~L}(F=4.3$, $p=0.041)$ and $\log _{10}$ hs-CRP $(F=6.8, p=0.011)$ were variables that interacted independently with Mean-IMT in all subjects. A stepwise multivariate regression analysis also demonstrated that $\mathrm{sCD} 40 \mathrm{~L}(F=13.6, p<0.001)$ and $\operatorname{sBP}(F=6.8, p=0.011)$ were variables that interacted independently with Max-IMT in all subjects (Table 3 ).

Additionally, serum sCD40L levels in patients without retinopathy $(n=53)$, with simple retinopathy $(n=21)$, and with proliferative retinopathy $(n=4)$ were $2.02 \pm 1.24,2.38 \pm$ 1.61 and $1.75 \pm 0.91 \mathrm{ng} / \mathrm{ml}$, respectively. There were no significant differences between the groups. There was no significant difference in serum SCD40L between patients with microalbuminuria or macroalbuminuria $(n=8,1.93 \pm$ $1.33 \mathrm{ng} / \mathrm{ml})$ and those without albuminuria $(n=68,2.13 \pm$ $1.35 \mathrm{ng} / \mathrm{ml})$. There was no correlation between serum SCD40L and daily urinary AER $(r=-0.041, p=0.726)$.

\section{Discussion}

Serum levels of $S C D 40 L$ are elevated in young patients with type 1 diabetes The results of this study indicate that serum SCD40L levels and carotid IMT were increased in subjects with type 1 diabetes and that there were statistically significant associations between sCD40L levels and carotid IMT.
In the present study, serum levels of sCD40L were higher in young type 1 diabetic patients than in agematched healthy controls, an observation consistent with previous reports $[13,14,20]$. Because there were differences in the baseline characteristics between controls and type 1 diabetes (especially in terms of sex, BMI and serum triglyceride), we performed a stepwise multivariate regression analysis that included type 1 diabetes, sex, BMI and serum triglyceride as independent variables, and found that the presence of type 1 diabetes was an independent determinant of a high SCD40L value. Although there was no significant association between $\mathrm{sCD} 40 \mathrm{~L}$ and $\mathrm{HbA}_{1 \mathrm{c}}$ in the present study, controversy remains as to whether there is a relationship between $\mathrm{sCD} 40 \mathrm{~L}$ and $\mathrm{HbA}_{1 \mathrm{c}}$ levels $[13$, $14,20,21]$. The current study also showed that serum levels of sCD40L were correlated with those of hs-CRP and that hs-CRP is an independent determinant of a high sCD40L value. Although our data do not permit any conclusions to be drawn as to whether the elevated SCD40L is a cause or a consequence of elevated hs-CRP, these results might reflect the pathophysiological relationship between inflammation and platelet hyperactivation in atherothrombosis [22].

Carotid IMT is associated with serum level of SCD40L It has been reported that increased levels of SCD40L are associated with atherosclerotic diseases [8-12]. Previous studies have also established carotid arterial wall thickness, IMT, as one of the most reliable markers of systemic atherosclerosis, both in subjects with and without diabetes [23]. However, the association between sCD40L levels 
and carotid IMT in subjects with type 1 diabetes has not been reported. To the best of our knowledge, this is the first report showing the relationship between subclinical, relatively early stage atherosclerosis and a marker of platelet hyperactivity (sCD40L) in subjects with type 1 diabetes. The present study has shown that carotid IMT is significantly greater in patients with type 1 diabetes than in control subjects and that there is a statistically significant association between sCD40L levels and carotid IMT. There was a significant association between hs-CRP and carotid IMT, linking inflammation to the development of atherosclerosis. Furthermore, hs-CRP was also linked to SCD40L, indicating an association between inflammation and thrombocyte activation. However, on multivariate analysis, sCD40 L remained an independent predictor of carotid IMT. Since type 1 diabetes is a major cause of cardiovascular morbidity and mortality, the results of the current study provide important information, suggesting that routine screening for carotid IMT could be important as a target for therapeutic interventions and monitoring to improve the clinical outcome in this high-risk population. However, this is a cross-sectional survey with a rather small number of subjects, and the association demonstrated between sCD40L levels and carotid IMT was statistically significant but not strong. Therefore, further study would be necessary to examine whether sCD40L actually accelerates the progression of atherosclerosis. Although sources of elevated SCD40L in diabetic patients will require further investigation, sCD40L may activate circulating leucocytes to enhance the release of proinflammatory cytokines, enhance the release of chemokines by mononuclear cells, and increase the expression of adhesion molecules at the endothelium $[8,19,24]$. These inflammatory responses may further promote the infiltration of activated leucocytes into the atherosclerotic lesion, which, in turn, may directly activate small mononuclear cells, macrophages and $\mathrm{T}$ cells inside the vessel wall, leading to thickening of the arterial wall [25]. Taking into account the pathological role of $\mathrm{SCD} 40 \mathrm{~L}$ reported in the previous studies, it would not merely be a marker but one of the key players in the progression of atherosclerosis [6$12,20,24,25]$.

In contrast to the significant association observed between SCD40L levels and carotid IMT, no significant difference in serum sCD40L levels was seen between individuals with and without diabetic microangiopathy (retinopathy and nephropathy). These results suggest that SCD40L levels are not closely associated with the progression of microangiopathy, although, because of the limited number of subjects with microangiopathy in the present study, we cannot exclude the possibility that SCD40L levels somehow influence the progression of microangiopathy. Further studies with larger study populations would be necessary to clarify this association.

In summary, serum levels of sCD40L and hs-CRP are elevated in young patients with type 1 diabetes, suggesting that platelet activity and inflammation are simultaneously accelerated in type 1 diabetes, even in younger generations. Furthermore, it is suggested that increased levels of serum sCD40L are associated with accelerated atherosclerotic changes observed in young patients with type 1 diabetes.

\section{References}

1. Schonbeck U, Libby P (2001) CD40 signaling and plaque instability. Circ Res 89:1092-1103

2. Ross R (1993) The pathogenesis of atherosclerosis: a perspective for the 1990s. Nature 362:801-809

3. Adams DH, Shaw S (1994) Leucocyte-endothelial interactions and regulation of leucocyte migration. Lancet 343:831-836

4. Lutgens E, Gorelik L, Daemen MJ, et al (1999) Requirement for CD154 in the progression of atherosclerosis. Nat Med 5: $1313-1316$

5. Mach F, Schonbeck U, Sukhova GK, Atkinson E, Libby P (1998) Reduction of atherosclerosis in mice by inhibition of CD40 signaling. Nature 394:200-203

6. Kiener PA, Moran-Davis P, Rankin BM, Wahl AF, Aruffo A, Hollenbaugh D (1995) Stimulation of CD40L with purified soluble gp39 induces proinflammatory responses in human monocytes. J Immunol 155:4917-4925

7. Hollenbaugh D, Mischel-Petty N, Edwards CP, et al (1995) Expression of functional CD40L by vascular endothelial cells. J Exp Med 182:33-40

8. Aukrust P, Muller F, Ueland T, et al (1999) Enhanced levels of soluble and membrane-bound CD40 ligand in patients with unstable angina: possible reflection of $\mathrm{T}$ lymphocyte and platelet involvement in the pathogenesis of acute coronary syndromes. Circulation 100:614-620

9. Garlichs CD, Eskafi S, Raaz D, et al (2001) Patients with acute coronary syndrome express enhanced CD40 ligand/CD154 on platelets. Heart 86:649-655

10. Tsakiris DA, Tschopl M, Wolf F, Labs KH, Jager KA, Marbet GA (2000) Platelets and cytokines in concert with endothelial activation in patients with peripheral arterial occlusive disease. Blood Coagul Fibrinolysis 11:165-173

11. Schonbeck U, Varo N, Libby P, Buring J, Ridker PM (2001) Soluble CD40L and cardiovascular risk in women. Circulation 104:2266-2268

12. Heeschen C, Dimmeler S, Hamm CW, et al (2003) Soluble CD40 ligand in acute coronary syndromes. N Engl J Med 348:1104-1111

13. Varo N, Vincent D, Libby P, et al (2003) Elevated plasma levels of the atherogenic mediator soluble CD40 ligand in diabetic patients. Circulation 107:2664-2669

14. Harding SA, Sommerfield AJ, Sarma J, et al (2004) Increased CD40 ligand and platelet-monocyte aggregates in patients with type 1 diabetes mellitus. Atherosclerosis 176:321-325

15. Yamasaki Y, Kawamori R, Matsushima H, et al (1994) Atherosclerosis in carotid artery of young IDDM patients monitored by ultrasound high-resolution B-mode imaging. Diabetes 43:634-639

16. Friedewald WT, Levy RI, Fredrickson DS (1972) Estimation of the concentration of low-density lipoprotein cholesterol in plasma, without use of the preparative ultracentrifuge. Clin Chem 18:499-502

17. Kawamori R, Yamasaki Y, Matsushima H, et al (1992) Prevalence of carotid atherosclerosis in diabetic patients: ultrasound high-resolution B-mode imaging on carotid arteries. Diabetes Care 15:1290-1294

18. Yamasaki Y, Kodama M, Nishizawa H, et al (2000) Carotid intima-media thickness in Japanese type 2 diabetic subjects: predictors of progression and relationship with incident coronary heart disease. Diabetes Care 23:1310-1315

19. Pignoli P, Tremoli E, Poli A, Oreste P, Paoletti R (1986) Intimal plus medial thickness of the arterial wall: a direct measurement with ultrasound imaging. Circulation 74:1399-1406 
20. Cipollone F, Chiarelli F, Davi G, et al (2005) Enhanced soluble CD40 ligand contributes to endothelial cell dysfunction in vitro and monocyte activation in patients with diabetes mellitus: effect of improved metabolic control. Diabetologia 48:1216-1224

21. Targher G, Zoppini G (2004) Soluble CD40L in young type 1 diabetic individuals without clinical microvascular and macrovascular complications. Diabetes Care 27:1236-1237

22. Andre P, Nannizzi-Alaimo L, Prasad SK, Phillips DR (2002) Platelet-derived CD40L: the switch-hitting player of cardiovascular disease. Circulation 106:896-899
23. O'Leary DH, Polak JF, Kronmal RA, et al (1992) Distribution and correlates of sonographically detected carotid artery disease in the Cardiovascular Health Study. Stroke 23:1752-1760

24. Noelle RJ (1996) CD40 and its ligand in host defense. Immunity 4: 415-419

25. Mach F, Schönbeck U, Bonnefoy J-Y, Pober JS, Libby P (1997) Activation of monocyte/macrophage functions related to acute atheroma complication by ligation of CD40: induction of collagenase, stromelysin, and tissue factor. Circulation 96:396-399 